\title{
AN OVERVIEW OF MILLIONSCHIKOV'S QUASI-NORMALITY HYPOTHESIS APPLIED TO TURBULENCE
}

\author{
C. MAMALOUKAS* \\ Department of Statistics \\ Athens University of Economics and Business \\ GREECE \\ E-mail: mamkris@aueb.gr \\ H.P. MAZUMDAR \\ Indian Statistical Institute \\ Calcutta - 700035, INDIA \\ E-mail: hpmi2003@yahoo.com
}

\begin{abstract}
In this paper, we examine the zero-fourth cumulant approximation that was applied to fluctuating velocity components of homogeneous and isotropic turbulence by M.D. Millionschikov. Since the publication of the remarkable paper of Millionschikov, many authors have applied this hypothesis to solve the closure problem of turbulence. We discuss here various studies by the other authors on the developments of this hypothesis and their applications to the incompressible velocity temperature, hydrodynamic and magnetohydrodynamic fluctuating pressure fields and the general magnetohydrodynamic turbulence field. Lastly, we discuss broadly the computational difficulties that arise in turbulence problems and their possible refinements. We include also some enlightments of the process of future work that could be undertaken in this field of research.
\end{abstract}

Key words: Millionschikov's quasi-normality hypothesis, homogeneous turbulence, isotropic turbulence, correlation tensors, zero-fourth cumulant approximation.

\section{Introduction}

The inherent difficulty with the closure problem of turbulence is well known. In tackling this problem, generally some physically based hypotheses are often constructed. Such a hypothesis was first forwarded by Chow (1940) and Millionschikov (1941) in which an assumption was made that the fourth order velocity correlation is related to the second order correlation in the same way as for a normal joint probability distribution of the velocity. A final version of this statistical hypothesis is named after M.D. Millionschikov. Uberoi (1953) conducted extensive measurements for some cases and showed that the discrepancy between the measured fourth order moments and the prediction deduced from Millionschikov's quasi-normality hypothesis are found to lie within the limits of experimental errors. Ogura (1963) carried out numerical computations of the turbulence energy spectrum $E(\kappa, t)$ and showed that it becomes negative over a finite wave number range when the Reynolds number becomes sufficiently large. Mirable (1969) pointed out that the negative energy observed by Ogura (1963) is due to the assumption that at the initial time $t=0$ the third order moment of the velocity field is identically zero.

\footnotetext{
* To whom correspondence should be addressed
} 
Millionschikov (1941) proposed for the decay of isotropic turbulence in its final stage that probability distributions of the velocities at two different points but at one and the same instant of time, are approximately in normal distribution. In effect, a relation between the fourth and second order moments is established but with their order moments differing from zero. Uberoi (1953) verified this hypothesis experimentally, in particular some of its essential consequences. It is known that triple correlation functions do not vanish in homogeneous turbulence. A theory however, is expected to be established without the vanishing of the third order correlations. Many scientists worked on the applicability of Millionschikov's hypothesis to more general cases of homogenous turbulence. Panchev (1971) reported that Millionschikov's hypothesis may be extended further to the cases wherein quadruple moments are formed for one and the same instant of time but for more than two points. Ogura (1963) discovered that the kinetic energy spectrum $E(\kappa, t)$ becomes negative over a finite wave number range if the Reynolds number is sufficiently large.

The quasi-normality hypothesis of Millionschikov in reference to four points, may be read as $\overline{u_{i} u_{j}^{\prime} u_{k}^{\prime \prime} u_{l}^{\prime \prime \prime}}=\overline{u_{i} u_{j}^{\prime}} \overline{u_{k}^{\prime \prime} u_{l}^{\prime \prime \prime}}+\overline{u_{i} u_{k}^{\prime \prime}} \overline{u_{j}^{\prime} u_{l}^{\prime \prime \prime}}+\overline{u_{i} u_{l}^{\prime \prime}} \overline{u_{j}^{\prime} u_{k}^{\prime \prime}}$. Proudman and Reid (1954) carried out investigations of the dynamic behavior of homogenous turbulence in which the joint probability distribution of fluctuations velocity components at three points is approximately normal. They showed that Loitsiansky's integral is not invariant of the motion and the first time derivative of the triple correlation function, say $K(r)$ is proportioned to for large values of $r$. In this approach, all velocity cumulants are exponentially small for large separations, which are local in their effect but they have values determined by the whole velocity field.

In order to remove the inconsistency of Proudman and Reid's approach, Batchelor and Proudman (1956) assumed essential kinds of asymptotic forms of velocity cumulants.

More specifically, according to them it is important to understand that the effect of pressure forces is subsequently to develop asymptotic forms that are integral power laws. They showed also that the velocity covariance $\overline{u_{i} u_{j}^{\prime}}$ reduces to the order of $r^{-5}$ when separation is large.

Mirabel (1969) pointed out that the negative energy, obtained by Ogura (1969) is the outcome of the assumption that at the initiation time $t=0$, the third moment of the velocity field is identically zero. This implies that at the initial time there is no transfer of energy over the velocity spectrum. One obvious suggestion is that at the initial situation, the third order moment need not be taken identically zero, instead it should be modeled differently. For example, at the initial stage, the eddies may be considered as the shape of cylindrical vortices, which are isotropic in the 2-dimensional plane normal to the axis of the cylinder. Ghosh (1972) investigated the early-period decay process of a general type of turbulence using quasi-normality hypothesis which when simplified for the case of ordinary isotropic turbulence yielded well known decay equation due to Proudman and Reid (1954). Ghosh established two lemmas to derive the limiting forms of the spectrum tensors and of Millionschikov's hypothesis when two or more points under reference coincide. These lemmas are used freely to derive Proudman-Reid style dynamical equations for the hydrodynamic velocity and temperature fields, hydromagnetic fields and relevant pressure-fluctuation fields, as arising in hydrodynamic and hydromagnetic turbulent flows. For convenience of the readers, we would briefly state the two lemmas here.

Lemma I: This is concerned with the behavior of correlation tensors in the energy space when two or more points under reference coincide.

Let $\Psi_{i, j}\left(\boldsymbol{\kappa}, \boldsymbol{\kappa}^{\prime}, t\right)$ and $\Psi_{i, j, \kappa}\left(\boldsymbol{\kappa}, \boldsymbol{\kappa}^{\prime}, \boldsymbol{\kappa}^{\prime \prime}, t\right)$ be spectrum functions which correspond respectively to $F_{i, j}\left(x, x^{\prime}, t\right)\left[=\overline{u_{i} u_{j}^{\prime}}\right]$ and $F_{i, j, \kappa}\left(x, x^{\prime}, x^{\prime \prime}, t\right)\left[=\overline{u_{i} u_{j}^{\prime} u_{\kappa}^{\prime \prime}}\right]$, as constructed by the fluctuating velocity components in the turbulent medium. 
Now when the third point $x^{\prime \prime}$ merges with the first point $x$, then we have the first lemma

$$
\int \Psi_{i, j, \kappa}\left(\lambda-\boldsymbol{\kappa}^{\prime \prime}, \boldsymbol{\kappa}^{\prime}, \boldsymbol{\kappa}^{\prime \prime}, t\right) d \boldsymbol{\kappa}^{\prime \prime}=\Psi_{i k, j}\left(\lambda, \boldsymbol{\kappa}^{\prime}, t\right)
$$

where

$$
\lambda=\kappa+\kappa^{\prime}
$$

Lemma II: It is concerned with Millionschikov's quasi-normality hypothesis. We consider an additional fluctuation velocity component $u_{l}^{\prime \prime \prime}$ at the point $x^{\prime \prime \prime}$, and the spectrum tensor $\Psi_{i, j, \kappa, l}\left(\kappa, \kappa^{\prime}, \kappa^{\prime \prime}, \kappa^{\prime \prime \prime}, t\right)$ which correspond to $F_{i, j, \kappa, l}\left(x, x^{\prime}, x^{\prime \prime}, x^{\prime \prime \prime}, t\right)\left[=\overline{u_{i} u_{j}^{\prime} u_{\kappa}^{\prime \prime} u_{l}^{\prime \prime \prime}}\right]$.

Now, when the fourth point $x^{\prime \prime \prime}$ coincides with the first point $x$, we derive the relation

$$
\begin{aligned}
& \Psi_{i l, j, \kappa}\left(\boldsymbol{\kappa}, \boldsymbol{\kappa}^{\prime}, \boldsymbol{\kappa}^{\prime \prime}, t\right)=\int \Psi_{i, j}\left(\mathbf{\kappa}-\boldsymbol{\kappa}^{\prime \prime \prime}, \boldsymbol{\kappa}^{\prime}, t\right) \Psi_{\kappa, l}\left(\boldsymbol{\kappa}^{\prime \prime}, \boldsymbol{\kappa}^{\prime \prime \prime}, t\right) d \mathbf{\kappa}^{\prime \prime \prime}+ \\
& +\int \Psi_{i, j}\left(\boldsymbol{\kappa}-\boldsymbol{\kappa}^{\prime \prime \prime}, \boldsymbol{\kappa}^{\prime \prime}, t\right) \Psi_{j, l}\left(\boldsymbol{\kappa}^{\prime}, \boldsymbol{\kappa}^{\prime \prime \prime}, t\right) d \boldsymbol{\kappa}^{\prime \prime \prime}+\int \Psi_{i l}\left(\boldsymbol{\kappa}^{\prime}, t\right) \Psi_{j, \boldsymbol{\kappa}}\left(\boldsymbol{\kappa}^{\prime}, \boldsymbol{\kappa}^{\prime \prime}, t\right) .
\end{aligned}
$$

Now, if in addition to merging of the fourth point with the first point, the third point merges with the second point, the following relation may be derived

$$
\begin{aligned}
& \Psi_{i l, j \kappa}\left(\boldsymbol{\kappa}^{\prime}, \boldsymbol{\kappa}^{\prime}, t\right)=\iint\left[\Psi_{i, j}\left(\boldsymbol{\kappa}-\boldsymbol{\kappa}^{\prime \prime \prime}, \boldsymbol{\kappa}^{\prime}-\boldsymbol{\kappa}^{\prime \prime}, t\right) \Psi_{\kappa, l}\left(\boldsymbol{\kappa}^{\prime \prime}, \boldsymbol{\kappa}^{\prime \prime \prime}, t\right)+\right. \\
& \left.+\Psi_{i, \kappa}\left(\boldsymbol{\kappa}-\boldsymbol{\kappa}^{\prime \prime \prime}, \boldsymbol{\kappa}^{\prime \prime}, t\right) \Psi_{j, l}\left(\boldsymbol{\kappa}^{\prime}-\boldsymbol{\kappa}^{\prime \prime}, \boldsymbol{\kappa}^{\prime \prime \prime}, t\right)\right] d \boldsymbol{\kappa}^{\prime \prime} d \mathbf{\kappa}^{\prime \prime \prime}+\Psi_{i l}(\boldsymbol{\kappa}, t) \Psi_{j, \kappa}(\boldsymbol{\kappa}, t) .
\end{aligned}
$$

In the next section, we would apply these lemmas to derive briefly the Proudman-Reid type decay equations for the velocity, temperature and hydromagnetic fields.

The relevant expressions for the fluctuation pressure fields under hydrodynamic and hydromagnetic conditions will also be stated for the sake of completeness.

\section{Applications of quasi-normality hypothesis}

\section{a) Early-period decay process of turbulence}

We state here briefly the derivation of the Proudman-Reid equation for the turbulence velocity field following Ghosh (1972). Ghosh (1972) carried out long calculations, constructing the spectral equations corresponding to the two-point and three-point velocity correlation functions and utilizing the lemmas concerning Millionschikov's quasi-normality hypothesis and derived the guiding equation for the early period decay process of turbulence as

$$
\frac{\partial^{2}}{\partial t^{2}} \Psi_{i, j}\left(\boldsymbol{\kappa}, \boldsymbol{\kappa}^{\prime}, t\right)=I_{l}\left(\boldsymbol{\kappa}, \boldsymbol{\kappa}^{\prime}, t\right)+I_{2}\left(\boldsymbol{\kappa}, \boldsymbol{\kappa}^{\prime}, t\right)
$$

The expressions for $I_{l}\left(\boldsymbol{\kappa}, \boldsymbol{\kappa}^{\prime}, t\right)$ and $I_{2}\left(\boldsymbol{\kappa}, \boldsymbol{\kappa}^{\prime}, t\right)$ are studied well in the work of Ghosh (1972). Ghosh (1972) showed that Eq.(2.1) is valid for the general type of turbulence stated above and represents the early-period decay process. 
Further, Ghosh showed that for the case of homogeneous turbulence Eq.(2.1) reduces to

$$
\frac{\partial^{2}}{\partial t^{2}} \Psi_{i, j}\left(\boldsymbol{\kappa}, \boldsymbol{\kappa}^{\prime}, t\right)=I_{l}\left(\boldsymbol{\kappa}, \boldsymbol{\kappa}^{\prime}, t\right)
$$

Now, Eq.(2.2) is implied under the assumption of homogeneity and isotropy, i.e., in view of the form for the second rank spectrum tensor $\Psi_{s, q}\left(\boldsymbol{\kappa}, \boldsymbol{\kappa}^{\prime}, t\right)$ as

$$
\Psi_{s, q}\left(\kappa,-\boldsymbol{\kappa}^{\prime}, t\right)=\frac{F(\kappa, t)}{4 \pi \kappa^{2}}\left\{\delta_{s q}-\frac{\kappa_{s} \kappa_{q}}{\kappa^{2}}\right\},
$$

to the well known Proudman-Reid decay equation

$$
\frac{\partial^{2}}{\partial t^{2}} \int_{0}^{\infty} 2 \kappa^{2} F(\kappa, t) d \kappa=\frac{4}{3}\left[\int_{0}^{\infty} \kappa^{2} F(\kappa, t) d \kappa\right]^{2}
$$

putting the form for $\overline{\omega^{2}}$, the mean square variation in one component of vorticity in the case of homogeneous and isotropic turbulence, as

$$
\frac{3}{2} \overline{\omega^{2}}=\int_{0}^{\infty} \kappa^{2} F(\kappa, t) d \kappa
$$

we may write Eq.(2.4) in the final form

$$
\frac{d^{2}}{d t^{2}} \overline{\omega^{2}}=\left(\overline{\omega^{2}}\right)^{2}
$$

\section{b) Decay process of turbulence at large Reynolds and Peclet numbers}

Mazumdar (1976) studied the fluctuating temperature field considered to be superimposed on a general field of eddy turbulence by employing the quasi-normality hypothesis. In this investigation Mazumdar approached phenomenologically that the region under consideration is such that the variations of the mean temperature and mean velocity may be neglected because the transportation of thermal energy from place to place is very rapid. Mazumdar followed a similar procedure as in section a) above and showed that the temperature energy spectrum $E_{\theta \theta}(\kappa, t)$ is governed by the decay equation under the condition of homogeneity and isotropy by the equation

$$
\frac{\partial^{2}}{\partial t^{2}} \int_{0}^{\infty} \kappa^{2} E_{\theta \theta}(\kappa, t) d \kappa=\frac{4}{3} \int_{0}^{\infty} \kappa^{2} E_{\theta \theta}(\kappa, t) d \kappa \cdot \int_{0}^{\infty} \kappa^{2} F(\kappa, t) d \kappa .
$$


The associated velocity field is governed by the Proudman-Reid Eq.(2.4). In obtaining these two decay equations quasi-normality hypothesis has been used freely.

It is to be noted that $E_{\theta \theta}(\kappa, t)$ corresponds to the correlation function $\overline{\theta \theta^{\prime}}\left(=F_{\theta, \theta}\left(x, x^{\prime}, t\right)\right)$.

c) Mazumdar (2010) derived the Proudman-Reid type decay equation for the magneto hydro-dynamic (MHD) turbulence when both the eddy Reynolds number and the magnetic Reynolds number are very large, as

$$
\begin{aligned}
& \frac{\partial^{2}}{\partial t^{2}} \int_{0}^{\infty} 2 \kappa^{2} F(\kappa, t) d \kappa=\frac{4}{3}\left[\int_{0}^{\infty} \kappa^{2} F(\kappa, t) d \kappa\right]^{2}+\frac{8}{3} \int_{0}^{\infty} \kappa^{4} G(\kappa, t) d \kappa \cdot \int_{0}^{\infty} G(\kappa, t) d \kappa+ \\
& -\frac{2}{3} \int_{0}^{\infty} \kappa^{4} F(\kappa, t) d \kappa \cdot \int_{0}^{\infty} G(\kappa, t) d \kappa, \\
& \frac{\partial^{2}}{\partial t^{2}} \int_{0}^{\infty} 2 \kappa^{2} G(\kappa, t) d \kappa=\frac{32}{5} \int_{0}^{\infty} \kappa^{2} F(\kappa, t) d \kappa \cdot \int_{0}^{\infty} \kappa^{2} G(\kappa, t) d \kappa+ \\
& +\frac{8}{3} \int_{0}^{\infty} \kappa^{4} F(\kappa, t) d \kappa \cdot \int_{0}^{\infty} G(\kappa, t) d \kappa-\frac{4}{3} \int_{0}^{\infty} \kappa^{4} G(\kappa, t) d \kappa \cdot \int_{0}^{\infty} G(\kappa, t) d \kappa .
\end{aligned}
$$
isotropy.

The set of Eqs (2.8) and (2.9) has been obtained under the assumption of homogeneity and

d) Mazumdar $(1979,1984)$ applied Millionschikov's quasi-normality hypothesis, respectively, to the fields of hydro-dynamic and hydro magnetic turbulence and derived the following expressions for the spectra of respective pressure fluctuations, as

$$
\overline{(\operatorname{grad} p)^{2}}=p^{2} \int_{0}^{\infty} \int_{0}^{\infty} F(\kappa, t) F\left(\kappa^{\prime}, t\right) \kappa \kappa^{\prime} \Psi\left(\frac{\kappa}{\kappa^{\prime}}\right) d \kappa d \kappa^{\prime},
$$

and

$$
\overline{(\operatorname{grad} \bar{\omega})^{2}}=\int_{0}^{\infty} \int_{0}^{\infty}\left\{F(\kappa, t) F\left(\kappa^{\prime}, t\right)+G(\kappa, t) G\left(\kappa^{\prime}, t\right)\right\} \kappa \kappa^{\prime} \Psi\left(\frac{\kappa}{\kappa^{\prime}}\right) d \kappa d \kappa^{\prime}
$$

where,

$$
\Psi(s)=\Psi\left(\frac{1}{s}\right)=-\frac{1}{8}\left(s^{3}-s^{-3}\right)+\frac{11}{24}\left(s-s^{-1}\right)+\frac{1}{16}\left(s-s^{-1}\right)^{4} \ln \frac{1+s}{|1-s|}
$$


It is to be noted that the expression for $\overline{(\operatorname{grad} p)^{2}}$, given in Eq.(2.10) is due to Heisenberg (1948), $\bar{\omega}$ is the total pressure, given by $\bar{\omega}=\frac{p}{\rho}+\frac{1}{2}|h|^{2}$, where $h$ is the magnetic field component, $G(\kappa, t)$ is the magnetic energy spectrum.

The solution of Proudman - Reid Eq.(2.6) is given by Monin and Yaglom (1975)

$$
\overline{\omega^{2}}=2^{\frac{2}{3}} \frac{\omega^{2}}{\omega_{1}^{\frac{1}{6}}} P(x ; 0,1), \quad x=\frac{2^{3}}{3}\left(\omega_{1}^{2}\right)^{\frac{1}{2}}\left(t-t_{l}\right)
$$

where $\overline{\omega_{1}^{2}}$ and $t_{1}$ are integration constants. $P(x ; 0,1)$ is the elliptic Weierstrass function.

For solving Eq.(2.7), it is put in the form

$$
\frac{d^{2} \overline{(\operatorname{grad} \theta)^{2}}}{d t^{2}}=\frac{2}{3} \overline{\omega^{2}} \cdot \overline{(\operatorname{grad} \theta)^{2}} .
$$

Solution of Eq.(2.14) is given by Monin and Yaglom (1975)

$$
\overline{(\operatorname{grad} \theta)^{2}}=\overline{\theta_{0}^{2}} P^{\prime}(x), \quad x=\frac{2^{\frac{1}{6}}}{3}\left(\omega_{0}^{2}\right)^{\frac{1}{2}}\left(t-t_{0}\right)
$$

where $P(x ; 0,1)$ is the elliptic Weierstrass function.

$\overline{\theta_{0}^{2}}, \overline{\omega_{0}^{2}}$ and $t_{0}$ are integration constants.

Calculations for variables in similar other problems may essentially be done in the same manner.

\section{Refinement of Millionschikov's quasi-normality hypothesis}

The applications of Millionschikov's quasi-normality hypothesis are found to fail in some cases. For example, in the case of convective boundary layer turbulence (Gryanik et al., 2005) found that the probability density functions of temperature and vertical velocity fluctuations are skewed.

They suggested closures for fourth-order moments which take skewness into account and obtained a very good agreement with the measured data. Losch (2004) devised parametrizations of fourth-order moments according to a universal model presented by Gryanik et al. (2005) and found them to be more accurate than their corresponding Gaussian parametrizations which are based on Millionschikov's hypothesis. The universal model is marginally improved by fitting the model parameters to the data.

For uniform homogeneous isotropic turbulence the system of moment equations, using Millionschikov's hypothesis, may be written in the form,

$$
\left(\frac{\partial}{\partial t}+2 v \kappa^{2}\right) E(\kappa, t)=\int_{0}^{\infty} \int_{\kappa-\kappa^{\prime}}^{\kappa+\kappa^{\prime}} F\left(\kappa, \kappa^{\prime}, \kappa^{\prime \prime}, t\right) d \kappa^{\prime \prime} d \kappa^{\prime},
$$


and

$$
\begin{aligned}
& {\left[\frac{\partial}{\partial t}+v\left(\kappa^{2}+\kappa^{\prime 2}+\kappa^{\prime \prime 2}\right)\right] F\left(\kappa, \kappa^{\prime}, \kappa^{\prime \prime}, t\right)=\phi_{1}\left(\kappa, \kappa^{\prime}, \kappa^{\prime \prime}\right) E(\kappa, t) E\left(\kappa^{\prime}, t\right)+} \\
& +\phi_{2}\left(\kappa, \kappa^{\prime}, \kappa^{\prime \prime}\right) E\left(\kappa^{\prime \prime}, t\right) E(\kappa, t)+\phi_{3}\left(\kappa, \kappa^{\prime}, \kappa^{\prime \prime}\right) E\left(\kappa^{\prime}, t\right) E\left(\kappa^{\prime \prime}, t\right)
\end{aligned}
$$

where $v$ is the kinematic viscosity,

$$
\begin{aligned}
& \kappa^{\prime \prime 2}=\kappa^{2}+\kappa^{\prime 2}+2 \mu \kappa \kappa^{\prime}, \\
& F\left(\kappa, \kappa^{\prime}, \kappa^{\prime \prime}\right)=8 \pi^{2} \kappa \kappa^{\prime} \kappa^{\prime \prime} \Gamma\left(\kappa, \kappa^{\prime}, \kappa^{\prime \prime}\right), \\
& \phi_{1}\left(\kappa, \kappa^{\prime}, \kappa^{\prime \prime}\right)=\left(\frac{q}{16 \kappa^{3} \kappa^{\prime 3} \kappa^{\prime \prime}}\right)\left(\kappa^{\prime 2}-\kappa^{2}\right)\left(\kappa^{\prime \prime 2}-\kappa^{2}-\kappa^{\prime 2}\right), \\
& \phi_{2}\left(\kappa, \kappa^{\prime}, \kappa^{\prime \prime}\right)=\left(\frac{q}{16 \kappa^{3} \kappa^{\prime} \kappa^{\prime \prime}}\right)\left[\kappa^{2}\left(\kappa^{\prime \prime 2}-\kappa^{2}+\kappa^{\prime 2}\right)-\kappa^{\prime \prime 2}\left(\kappa^{\prime \prime 2}-\kappa^{2}-\kappa^{\prime 2}\right)\right], \\
& \phi_{3}\left(\kappa, \kappa^{\prime}, \kappa^{\prime \prime}\right)=\left(\frac{q}{16 \kappa \kappa^{\prime 3} \kappa^{\prime \prime 3}}\right)\left[\kappa^{\prime \prime 2}\left(\kappa^{\prime \prime 2}-\kappa^{2}-\kappa^{\prime 2}\right)-\kappa^{\prime 2}\left(\kappa^{\prime \prime 2}+\kappa^{2}-\kappa^{\prime 2}\right)\right], \\
& q=4 \kappa^{2} \kappa^{\prime 2}-\left(\kappa^{\prime \prime 2}-\kappa^{2}-\kappa^{\prime 2}\right)^{2} .
\end{aligned}
$$

The expressions for the energy spectrum $E(\kappa)$ and the spectral form of the third-order velocity-field are written as

$$
E(\kappa)=4 \pi \frac{\sigma}{a^{4}} \kappa^{4} \exp \left(-\frac{\kappa^{2}}{a^{2}}\right)
$$

and

$$
F_{i j l}\left(\boldsymbol{\kappa}, \boldsymbol{\kappa}^{\prime}\right)=i \frac{\sigma}{a^{4}} \varepsilon_{i m}\left(\kappa_{m}+\kappa_{m}^{\prime}\right) \times\left(\boldsymbol{\kappa} \boldsymbol{\kappa}^{\prime} \delta_{j l}-\kappa_{l} \kappa_{j}^{\prime}\right) \exp \left(-\frac{\kappa^{2}+\kappa^{\prime 2}+\kappa \boldsymbol{\kappa}^{\prime}}{a^{2}}\right) .
$$

The spectral tensor $F_{i j l}\left(\boldsymbol{\kappa}, \boldsymbol{\kappa}^{\prime}\right)$ which is based on the assumption of the scalar function $i \kappa_{l} F_{l j j}\left(\kappa, \boldsymbol{\kappa}^{\prime}\right)$, has the following form in the present case 


$$
\begin{aligned}
& \Gamma\left(\kappa, \kappa^{\prime}, \mu\right)=i \kappa_{l} F_{l j j}\left(\kappa, \kappa^{\prime}\right)=\frac{\sigma}{a^{4}} \operatorname{sign}\left(\kappa^{\prime}-\kappa\right) \mu \kappa^{2} \kappa^{\prime 2}\left(1-\mu^{2}\right)^{\frac{1}{2}} \times \exp \left(-\frac{\kappa^{2}+\kappa^{\prime 2}+\mu \kappa \kappa^{\prime}}{a^{2}}\right), \\
& \left(\mu=\frac{\kappa \kappa^{\prime}}{\kappa \kappa^{\prime}}\right) .
\end{aligned}
$$

Mirabel (1969) solved numerically the equation for the kinetic energy spectrum (3.1) based on a finite difference method with the initial conditions (3.3) and (3.4).

Mirabel (1969) described the evolution of the energy spectrum for initial zero (Ogura, 1963) and non-zero third moments in his paper. Such descriptions were found reasonable at the initial stage but after some time a contradiction was found to arise. On the basis of the above analysis, we may arrive at the following conclusions.

\section{Conclusions}

i) Millionschikov's quasi-normality hypothesis is considered to be very useful for closure of homogeneous and isotropic turbulence as such a hypothesis has been proved to be valid within the limits of experimental errors.

ii) To gain more insight into the closure problems of turbulence, data from recent and advanced level measurements are to be brought into account in developing appropriate models.

iii) As and when necessary, modification of this hypothesis is welcome, as Ogura (1963) has pointed tentatively that the errors that arise from finite difference approximations in numerical integration of the main equation of turbulence are not responsible for the generation of the negative energy spectrum but are the consequences of the quasi-normality hypothesis itself.

iv) Third-order moments of the velocity field should not be assumed zero and it should be taken into account into the calculation even at the initial evolution of spectral energy of turbulence.

v) It is to be noted that the measured values of fourth-order mixed velocity-temperature moments in the atmospheric surface layer agree well with the quasi-gaussian assumption. Further, Schwarz inequalities, commonly used in the clipping approximation in turbulence modeling, are found to provide counts for third-order moments of $\omega, \theta$ that are too conservative. These types of approaches are to be encouraged.

\section{Nomenclature}

$$
\begin{aligned}
E(\kappa) & - \text { energy spectrum } \\
E_{\theta \theta}(\kappa, t) & - \text { isotropic temperature energy spectrum function } \\
F(\kappa, t) & - \text { isotropic energy spectrum function } \\
F_{i j l}\left(\kappa, \kappa^{\prime}\right) & - \text { spectral tensor } \\
G(\kappa, t) & - \text { magnetic energy spectrum } \\
h & - \text { magnetic field component } \\
P(x ; 0,1) & - \text { eliptic Weierstrass function } \\
p & - \text { fluctuating pressure components } \\
u_{i} & - \text { turbulent component of the velocity field } \\
\overline{u_{i} u_{j}^{\prime}} F_{i, j}\left(x, x^{\prime}, t\right) & - \text { two-points correlation function of turbulent velocity components } \\
\overline{u_{i} u_{j}^{\prime} u_{k}^{\prime \prime}} F_{i, j, \kappa}\left(x, x^{\prime}, x^{\prime \prime}, t\right) & - \text { three-points correlation function of turbulent velocity components }
\end{aligned}
$$




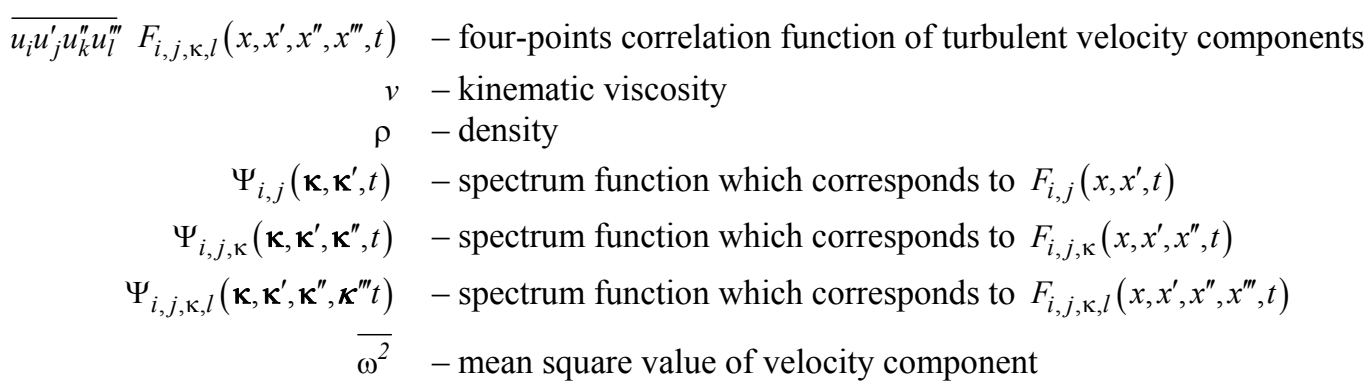

\section{References}

Antonia R.A., Chambers A.J. and Bradley E.F. (1982): Third- and fourth-order mixed moments of turbulent velocity and temperature fluctuations in the atmospheric surface layer. - Boundary Layer Meteorology. - vol.22, pp.421430.

Batchelor G.K. and Proudman I. (1956): The large-scale structure of homogeneous turbulence. - Phil. Trans. Roy. Soc., vol.248A, p.167.

Canuto V.M. (1992): Turbulent convection with overshooting: Reynolds stress approach. - Astrophys. J., vol.392, pp.218-232.

Chow W.L. (1940): Über Systeme von linearen partiellen differentialgleichungen erster ordnung. - J. Math. Ann., pp.98-105.

Ghosh K.M. (1972): Some concequences of Millionschikov's hypothesis in the early-period decay process of turbulence. - Indian Journal of Pure and Appl. Math., vol.3, No.1, p.157.

Gryanik V.M., Hartmann J, Raasch S. and Schröter M. (2005): A Refinement of the Millionshchikov quasi-normality hypothesis for convective boundary layer turbulence. - J. Atmos. Sci., vol.62, pp.2632-2638.

Heisenberg (1948): On the theory of statistical and isotropic turbulence. - Proceedings of the Royal Society of London. Series A, Mathematical and Physical Sciences vol.195, No.1042, pp.402-406.

Losch M. (2004): On the validity of the Millionshchikov quasi-normality hypothesis for open-ocean deep convection. Geophysical Research Letters, vol.31, L23301, No.4 pp., Doi:10.1029/2004GL021412.

Mazumdar H.P. (1976): On the decay process of turbulence at large Reynolds and Peclet numbers. - App. Sci. Res., vol.32, p.571.

Mazumdar H.P. (1979): On the pressure fluctuations associated with a general type of turbulence. - Appl. Sci. Res. vol.35, No.5-6, pp.367-371.

Mazumdar H.P. (1984): On the fluctuations of total pressure associated with general type of hydromagnetic turbulence. - Archeves of Mechanics, vol.36, pp.233-240.

Mazumdar H.P. (2010): On Incompressible Hydromagnetic Turbulence. (Research Monograph). - Calcutta Mathematical Society.

Mazumdar H.P. and Mandal B.C. (2009): On persen theory of two dimensional turbulent boundary layer. - Int. J. of Appl. Mech. and Engineering, vol.14, No.4, pp.1009-1028.

Millionschikov M. (1941): On the theory of homogeneous isotropic turbulence. - Dokl. Akad. Nauk SSSR, vol.32, pp.615-618.

Mirabel A.P. (1969): Application of Millionschikov's hypothesis to the problem of isotropic turbulence degeneration.Izv. AN SSSR. Mekhanika Zhidkosti i Gaza, vol.4, No.5, pp.171-175.

Monin A.S. and Yaglom A.M. (1975): Statistical Fluid Mechanics. - Vol. II, MIT Press, Cambridge, MA. 
Ogura Y. (1963): A consequence of the zero-fourth-cumulant approximation in the decay of isotropic turbulence. Journal of Fluid Mechanics, vol.16, pp.33-40.

Panchev S. (1971): Random functions and turbulence. - Oxford: Pergamon Press., p.444.

Proudman I. and Reid W.H. (1954): On the decay of a normally distributed and homogeneous turbulent velocity field. Phil. Trans. Roy. Soc. vol.247A, p.163-189.

Uberoi M.S. (1953): Quadruple velocity correlations and pressure fluctuations in isotropic turbulence. - J. Aeronaut. Sci., vol.20, p.197.

Received: December 3, 2012

Revised: August 8, 2013 